\title{
Replicability Challenges in Location Analytics
}

\author{
Alan T. Murray \\ Department of Geography \\ University of California at Santa Barbara \\ amurray@ucsb.edu
}

\begin{abstract}
The advances in GIS capabilities have been significant over the past few decades, with commercial and open-source software providing relatively easy access to location analytics for even the most novice of users. While many computational methods are indeed accessible and widely applied, this paper focuses on spatial optimization based location analytics available through GIS because of their increased adoption to address a range of social and environmental issues. The significance of this is that insights, management, planning, decision making and policy results are informed by location analytics. Important questions arise, however, about appropriateness, assumptions and validity, especially when user-friendly point-and-click software is involved. Replicability is at the heart of concerns when social and environmental issues are addressed using GIS-based location analytics. Much interest has been devoted to data uncertainty, frame dependency, modifiable areal unit problem and the theoretical assumptions of developed methods, but little attention has been given to definition and implementation details for many advanced location analytics that can be found in GIS. This paper explores these issues as commercial and open-source GIS software incorporate a wide range of optimization based location analytics may face challenges in being replicable, reliable or reproducible.
\end{abstract}

\section{Introduction}

The extent and depth of analysis possible using modern geographic information systems (GIS) is remarkable, particularly considering their evolution over the past few decades. Not only are commercial and open-source GIS software user-friendly, enabled by point-and-click interfaces, but there exists libraries in Python and $\mathrm{R}$ that facilitate access to and extension of GIS functions as well. In many respects, these are exciting times for the use of GIS in deriving insights, management planning, decision making and policy evaluation/formulation involving environmental and socio-economic systems. There are in fact many reported studies in the literature where advanced location analytics in GIS have been featured. Select applications are included in Table 1. These studies represent location intelligence approaches, relying on GIS to access integrated optimization methods. What is noteworthy is that the academic contribution in these instances is the substantive application, not analytic development or refinement. Evident then is the significant increase in recent location intelligence studies relying on GIS-based spatial optimization, particularly those relying on commercial GIS packages.

Given the extent of analysis possible, it is natural to reflect on the practical implications of location analytical capabilities available through GIS. Indeed, researchers in GIS and spatial analysis have long undertaken such reflection. Work on the modifiable areal unit problem [29], frame dependence [35] and data uncertainty [13] are but a few examples of critical assessment that can be noted.

Of course, reflection along these lines is very much part of broader scientific concerns regarding the validity of insights gained from analytics. Much discussion can be found in the academic literature, with a summary and overview found in [28]. More specific to GIS and spatial analytics, $[5,30,16]$ offer perspective and context regarding the significance of reflection. Two prevailing concepts are reproducibility and replicability. Reproducibility has to do with an ability to independently obtain/verify reported findings when the same data inputs are used. Replicability suggests an ability to repeat an approach using new data. [16] provide summary, suggesting that an analytical method is reproducible if independently used data plus methods give the same results and replicable if method results are similar using new data. These concepts generalize the previously mentioned concerns raised in the context of modifiable areal unit problem, frame dependence, data uncertainty, etc. that have long existed in location analytics. 
Table 1 Location intelligence studies.

\begin{tabular}{|c|c|c|}
\hline Reference & Context & Outlet \\
\hline [10] Costa et al. (2020) & Bioenergy plants & Renewable Energy \\
\hline [3] Banerjee et al. (2020) & Bikeshare stations & $\begin{array}{l}\text { Transportation Research } \\
\text { Interdisciplinary Perspectives }\end{array}$ \\
\hline [2] Athira et al. (2020) & Cement plants & $\begin{array}{l}\text { Journal of Material Cycles and } \\
\text { Waste Management }\end{array}$ \\
\hline [19] Lane et al. (2020) & $\begin{array}{l}\text { Hydrogen refueling } \\
\text { stations }\end{array}$ & Applied Energy \\
\hline [12] Gomez et al. (2019) & Trauma centers & Injury \\
\hline [22] Mishra et al. (2019) & $\begin{array}{l}\text { Community health cen- } \\
\text { Ters }\end{array}$ & Journal of Transport Geography \\
\hline [20] Lemire et al. (2019) & Biomass depots & $\begin{array}{l}\text { Biofuels, Bioproducts and } \\
\text { Biorefining }\end{array}$ \\
\hline $\begin{array}{l}\text { [33] Schroder et al. } \\
\text { (2019) }\end{array}$ & Transport routes & $\begin{array}{l}\text { Computers, Environment and Urban } \\
\text { Systems }\end{array}$ \\
\hline $\begin{array}{l}{[18] \text { Kocatepe et al. }} \\
(2018)\end{array}$ & Pet shelters & $\begin{array}{l}\text { International Journal of Disaster } \\
\text { Risk Reduction }\end{array}$ \\
\hline $\begin{array}{l}\text { [15] Irannezhad et al. } \\
(2018)\end{array}$ & Shipping routes & Transportation Research D \\
\hline $\begin{array}{l}\text { [31] Romanillos and } \\
\text { García-Palomares (2018) }\end{array}$ & & $\begin{array}{l}\text { Journal of Urban Planning and } \\
\text { Development }\end{array}$ \\
\hline [1] Alho et al. (2018) & Freight loading bays & Transportation Research D \\
\hline [11] Fraser et al. (2018) & Public cooling centers & Urban Climate \\
\hline [17] Khan et al. (2018) & Solid waste facilities & Science of The Total Environment \\
\hline $\begin{array}{l}\text { [37] Trindade et al. } \\
(2018)\end{array}$ & Evacuation routes & Natural Hazards \\
\hline $\begin{array}{l}{[34] \text { Teixeira et al. }} \\
(2018)\end{array}$ & Biomass power plants & Biomass and Bioenergy \\
\hline [4] Blanco et al. (2018) & $\begin{array}{l}\text { Plastic waste collection } \\
\text { center }\end{array}$ & $\begin{array}{l}\text { Resources, Conservation and } \\
\text { Recycling }\end{array}$ \\
\hline
\end{tabular}

[25] outlines important aspects of uncertainties in location analytics, including specification, representation, implementation and interpretation. A reasonable attempt to classify sources of geographic uncertainty associated with the scientific method process is offered in [16], where conceptual, measurement, analytical and communication capture many of the types of issues encountered. Two distinctions that do not fit within these summaries are based on problem definitions and actual users. Highlighted in [24] is that location analytics can be defined in different ways. That is, even geographic notions that are commonly understood concepts can be interpreted differently. For example, [24] reviews the "center", demonstrating that different modeling assumptions and conditions reflect different spatial processes. The result is the identification of a different geographic center. Location intelligence can be subjective, and impacted in various ways. This creates challenges for contemporary GIS as users may have little technical knowledge about the methods they are applying. The novice-expert distinction is particularly salient for advanced location analytics as GIS provides easy access through user-friendly interfaces that are inherently point-and-click. There is little doubt that part of the success and appeal of GIS is due to ease of access, making it critically important and relevant in the environmental and social sciences. [26] highlights that many different location analytic functions are accessible in GIS, as are many other advanced spatial analytics. As a result, spatial optimization approaches are popular among users. Indeed, they have been applied to address a wide range of social and environmental problems, as reflected in the studies noted in Table 1 (see also [27, 38]).

This paper discusses user knowledge along with method definition. This is done in the context of spatial optimization / location analytics available through GIS. Issues of specification, parameterization, solution and tradeoffs are explored. The next section reviews spatial optimization. This is followed by extension efforts possible associated with multiple objective models. Replicability implications are then reviewed. The paper ends with discussion and concluding comments.

\section{Spatial Optimization}

There is a rich literature on spatial optimization as an independent specialty domain, but its role within the context of GIS may well be even more significant. Spatial optimization underlies many advanced spatial analytical methods in one way or another. Reviews by $[7,36]$ offer a broad context and defining characteristics of spatial optimization. [23, 26] detail how spatial optimization is present throughout GIS analytical functions.

A definition of spatial optimization is that it involves structuring and solving a problem to identify the best decisions that conform to restrictions, with variables, coefficients and/or equations being geographic in some way, explicitly or implicitly. Google Scholar indicates that there are 3,110,000 documents that mention spatial optimization. A search of "spatial optimization" gives 8,860 documents. While not broken down here, the growth by year of these particular documents is exponential. This suggests increased importance and significance, and helps to explain why more GIS-based spatial optimization applications are appearing in the literature in recent years (see Table 1).

A spatial optimization problem may be stipulated in many different ways, including descriptions, flowcharts, code and mathematical models [25]. The most precise and unambiguous approach is as a mathematical model. Consider the following notation:

$i=$ index of demand areas (entire set $I$ )

$j=$ index of potential facility sites (entire set $J$ )

$a_{i}=$ service demand in area $i$

$N_{i}=$ set of facility sites capable of serving demand $i$ within distance or travel time standard

$p=$ number of facilities to be sited

These are model coefficients and parameters, all known or derived in advance of application of the spatial analytic method. The decision variables, or unknowns, are the following: 
$Y_{i}=\left\{\begin{array}{l}1 \text { if service coverage provided to demand } i \\ 0 \text { otherwise }\end{array}\right.$ $X_{j}=\left\{\begin{array}{l}1 \text { if facility located at potential site } j \\ 0 \text { otherwise }\end{array}\right.$

The significance of spatial optimization is that it can be used to formalize many location analytic approaches relied upon in the context of location intelligence. The notation then stipulates the demand to be served, $i$ (and expected amount, $a_{i}$ ), the potential sites for service facilities $j$ and number of facilities necessary, $p$. The decision variables are inherently spatial, indicating where to locate a facility, $X_{j}$, and which demand are covered, $Y_{i}$. Additionally, the set for each demand, $N_{i}$, identifies which potential facility locations could provide suitable service. An inherent capability of GIS is managing layers of information that serve as model inputs, such as demand areas to be served, suitability analysis associated with specification of potential facility sites, and deriving spatial service sets like $N_{i}$, often based on distance or travel time between two locations [9]. The coefficients can be combined with the decision variables to reflect a model or method of interest. A representative location analytic approach accessible through commercial GIS is the following:

$$
\begin{array}{ll}
\text { Maximize } & \sum_{i \in I} a_{i} Y_{i} \\
\text { Subject to } & \sum_{j \in N_{i}} X_{j}-Y_{i} \geq 0 \forall i \in I \\
& \sum_{j \in J} X_{j}=p \\
& X_{j} \in\{0,1\} \forall j \in J \\
& Y_{i} \in\{0,1\} \forall i \in I
\end{array}
$$

The objective (1) indicates the intent to maximize demand covered. Constraints (2) link location selection decisions to the coverage of demand. Constraint (3) specifies the number of facilities to be sited. Binary conditions are imposed in constraints (4) and (5).

This mathematical model, (1)-(5), describes a problem that has been vital in the social and environmental sciences, involving the need to locate a pre-specified number of service facilities in order to respond to demand distributed throughout a region. This is known as the maximal covering location problem [6,9]. [8] indicate that this model is available in commercial GIS as an advanced location analytic, readily applied in practice. As a result, it has indeed been relied upon for broad application to address a range of social, environmental and economic problems / issues as reflected in Table 1 and discussed in [8, 27].

Of course, as noted above, there are in fact a variety of ways to express a location analytic approach (e.g., descriptions, flowcharts, code and mathematical models). [27] discuss the "MAXIMIZE_COVERAGE" location analytic description found in ArcGIS: "This option solves the fire station location problem. It chooses facilities such that all or the greatest amount of demand is within a specified impedance cutoff." While ambiguous, it does correspond to the above model, (1)-(5). This location analytic approach can be accessed in ArcGIS through ArcToolbox, under the Network Analyst Tool. With data layers and input parameters, solution of the model is initiated and results are automatically displayed in ArcGIS. This model can also be found in TransCAD, described along the lines found in ArcGIS [27].

In general terms, there are different ways to solve a spatial optimization problem. The two prominent distinctions are exact and heuristic. An exact approach is one that can establish and prove that an identified solution is optimal. That is, there are conditions under which it can be definitively concluded that there is no better solution possible. An exact approach may therefore deem a solution as optimal or establish provable bounds on solution quality. A heuristic, on the other hand, is an approach or procedure that solves an optimization problem, but cannot establish meaningful solution quality. A heuristic may or may not identify a good or feasible solution. Often heuristics are computationally efficient, capable of fast solution times. This does not mean that the solution is of high quality, however. The important point is that a heuristic may be convenient and useful, but it does carry with it the caveat that there is no certainty about how good (or bad) the identified solution may be.

Recent work by [27, 38] discuss that GIS software packages like ArcGIS and TransCAD solve spatial optimization problems using a heuristic, providing users with results that are of unknown and unproven quality. This is true for "MAXIMIZE_COVERAGE", (1)-(5), in ArcGIS. The solution heuristic is described as [27]: “... location-allocation solver starts by generating an origin-destination matrix of shortestpath costs between all the facilities and demand point locations along the network ... then generates a set of semirandomized solutions and applies a vertex substitution heuristic (Teitz and Bart) to refine these solutions creating a group of good solutions. A metaheuristic then combines this group of good solutions to create better solutions. When no additional improvement is possible, the metaheuristic returns the best solution found. The combination of an edited matrix, semirandomized initial solutions, a vertex substitution heuristic, and a refining metaheuristic quickly yields near-optimal results." TransCAD too relies on a heuristic solution approach, though no descriptive details are provided. In both 
cases, as noted above, the heuristic offers no guarantee of solution quality, and no mechanism to quantify or characterize an identified solution for (1)-(5).

\section{Extending Location Analytics}

In support of location intelligence, there are clearly replicability challenges with GIS-based location analytics. More discussion focuses on this in the sections that follow. However, through standard GIS data processing and manipulation, it is possible to extend many of the location analytics found in current software. The "MAXIMIZE COVERAGE" in ArcGIS is an interesting example of this. As reviewed previously, (1)-(5) represents a clear and explicit specification of the actual location analytic approach. Consider the introduction of a second demand measure, $\hat{a}_{i}$, for each area $i$. Suppose that $a_{i}$ is the nighttime demand for service, whereas $\hat{a}_{i}$ is the daytime demand for service. That is, there is a diurnal shift, where $a_{i}$ represents demand assuming residential origins for service and $\hat{a}_{i}$ reflects a work based origin (daytime) for service. Of course, this is essentially a delineation of two distinct demand periods for a day and could be extended to many periods. Without loss of generality, the focus here is limited to two periods. There are implications for service provision, and this would need to be reflected in the associated spatial optimization model. In this particular case, (1)-(5) could be extended to account for multiple demand types through the use of multiple objectives.

The formulation for an extended version of the maximal covering location problem is:

$$
\begin{array}{ll}
\text { Maximize } & \sum_{i \in I} a_{i} Y_{i} \\
\text { Maximize } & \sum_{i \in I} \hat{a}_{i} Y_{i} \\
\text { Subject to } & \sum_{j \in N_{i}} X_{j}-Y_{i} \geq 0 \quad \forall i \in I \\
& \sum_{j \in J} X_{j}=p \\
& X_{j} \in\{0,1\} \forall j \in J \\
& Y_{i} \in\{0,1\} \forall i \in I
\end{array}
$$

The first objective (6) seeks to cover the maximum demand $\left(a_{i}\right)$ possible. The second objective (7) seeks to cover the maximum demand possible using an alternative measure of service demand, $\hat{a}_{i}$. Again, one could consider $a_{i}$ nighttime demand for service and $\hat{a}_{i}$ daytime demand for service. Constraints (8) link location selection decisions to coverage of demand. Constraint (9) specifies the number of facilities to be sited. Binary conditions are imposed in constraints (10)-(11). The model therefore retains much of the form and structure as originally stipulated in (1)-(5), but an additional objective is incorporated to account for an alternative demand consideration.

This particular model variant, (6)-(11), has been referred to as the multiobjective facility location problem in [32], and also has been utilized for addressing various substantive applications, including telecommunication access [14]. Multiobjective (or in this case biobjective) optimization models are more challenging to solve, either exactly or heuristically. A discussion and review addressing a biobjective problem can be found in [21]. In general, one is potentially interested in all Pareto-optimal or nondominated solutions. Given a feasible solution, it is non-dominated if no improvement is possible for one objective without degrading the other objective. The challenge, however, is identifying such solutions as this is not a trivial task.

In a GIS environment, one could consider the following simplification of objectives (6) and (7):

Maximize $\sum_{i \in I} w a_{i} Y_{i}+\sum_{i \in I}(1-w) \hat{a}_{i} Y_{i}$

where $w \in[0,1]$ is a weighting preference, relating objectives (6) and (7) as a linear function. This is known as the weighting method for solving multiobjective optimization problems. Objective (12) can be simplified mathematically as:

Maximize $\sum_{i \in I}\left[w a_{i}+(1-w) \hat{a}_{i} Y_{i}\right]$

Consider the following:

$\tilde{a}_{i}=w a_{i}+(1-w) \hat{a}_{i}$

where $\tilde{a}_{i}$ is simply the weighted combination of the two different demand estimates for area $i$. Given a particular weight $w, \tilde{a}_{i}$ is known and could be substituted for $a_{i}$ in objective (1) of the original model formulation. Doing so would mean that this extended model could be structured and solved in ArcGIS as "MAXIMIZE_COVERAGE" (or in TransCAD as well). If methods like noninferior set estimation or an enumeration based search of different values of $w$ are considered (possibly hundreds, thousands, millions or more), then an approximate non-dominated tradeoff frontier could be identified, perhaps along the lines of that shown in Figure 1. 


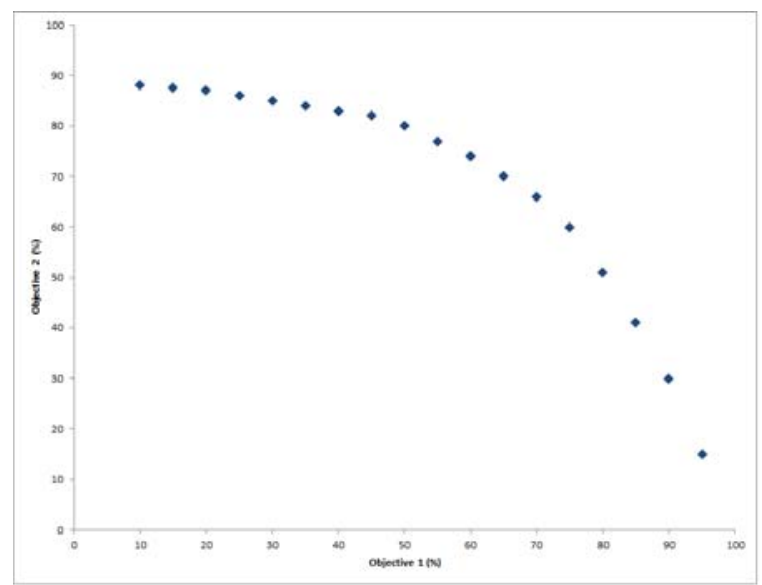

Figure 1. Biobjective tradeoff frontier summarizing non-dominated solutions.

Table 2. Objective values summarized in Figure 1.

\begin{tabular}{|l|c|c|}
\hline Solution & Objective 1 & Objective 2 \\
\hline 1 & 95 & 15 \\
\hline 2 & 90 & 30 \\
\hline 3 & 85 & 41 \\
\hline 4 & 80 & 51 \\
\hline 5 & 75 & 60 \\
\hline 6 & 70 & 66 \\
\hline 7 & 65 & 70 \\
\hline 8 & 60 & 74 \\
\hline 9 & 55 & 77 \\
\hline 10 & 50 & 80 \\
\hline 11 & 45 & 82 \\
\hline 12 & 40 & 83 \\
\hline 13 & 35 & 84 \\
\hline 14 & 30 & 85 \\
\hline 15 & 25 & 86 \\
\hline 16 & 20 & 87 \\
\hline 17 & 15 & 87.5 \\
\hline 18 & 10 & 88 \\
\hline
\end{tabular}

What is the point? GIS is regularly used in this way, to combine feature/object attributes in linear, non-linear and other ways. The linear combination in (14), and many similar to it, are often carried out in GIS-based application. An issue with the weighting method, however, is that it may not be capable of finding all non-dominated solutions in the case of integer restricted decision variables. That is, there may be non-dominated solutions in the so called gap regions of the Pareto tradeoff frontier [21]. This is particularly true for "MAXIMIZE_COVERAGE" due to the binary integer requirements, (10) and (11), meaning that all actual non-dominated solutions likely cannot be found using the weighting method. Of course, there is the issue of using a heuristic as well, suggesting that all (most?) solutions are not actually non-dominated since they are not optimal. Thus, at best the use of "MAXIMIZE_COVERAGE" enables only an approximation of non-dominated solution tradeoffs.

\section{Replicability Challenges}

As noted previously, reproducibility and replicability are increasingly important concepts. Location analytics involving spatial optimization through GIS poses a real need for more attention, especially what method is being applied and what is known (and not known) about any obtained results. How can reported findings be independently verified if the method is only vaguely described? How can the approach be replicated without a clear understanding of the method and solution approach?

For location analytics in ArcGIS, namely (1)-(5), "MAXIMIZE_COVERAGE" initiates a heuristic solution routine. The obtained results may be of high quality, or may not be. Murray et al. (2019) report that about $50 \%$ of the over 1,000 problem instances solved were found to be sub-optimal. Xu et al. (2020) report $63 \%$ of the 180 problem instances were sub-optimal for the captivated version of "MAXIMIZE_COVERAGE". Similar results were found using TransCAD. Empirical evidence suggests that the observed solutions generally appear to be within $10 \%$ of optimality, but that does not make them either reproducible or replicable. Perhaps an even more important and pressing issue is whether the observed results are actually reliable in some way. When coverage reflects suitable emergency response, such as that associated with fire station siting, then this equates to protection of property and lives. Failure to achieve the best possible outcome means that lives would be lost, property losses higher, etc. than would be possible if the optimal service configuration is employed. Application context would therefore be critical for making such an assessment of reliability. The implication is that heuristically obtained results may be reliable in some contexts but perhaps not in others.

Issues of appropriateness and validity as well as underlying assumptions are further complicated by extensions of advanced location analytics. It is indeed possible to address multiobjective situations using "MAXIMIZE_COVERAGE", formalized in (6)-(11). As noted, a common approach is through the linear combination structured in (13) as this may be easily implemented using (14). In practice, however, only one value of $w$ is often considered. This amounts to only one non-dominated solution being found in Figure 1, assuming it is optimal, when indeed there likely are many non-dominated solutions. Again, the implications for reproducibility and replicability are significant, as short of identifying all actual non- 
dominated tradeoff solutions, then a general understanding and characterization is not in fact possible.

To more explicitly highlight replicability challenges along these lines, consider the U.S. Special Supplemental Nutrition Program for Women, Infants, and Children in Santa Barbara County, providing nutrition information, healthy foods, breastfeeding education and health care services across the region. Location analytics were used to examine issues of access and accessibility, and in particular the maximal covering location problem was applied to identify and evaluate service center configuration design that takes into account daytime and nighttime population distributions.

Table 3. Identified solutions using ArcGIS.

\begin{tabular}{|l|c|c|}
\hline $\boldsymbol{w}$ & $\begin{array}{c}\text { Objective 1 } \\
\text { (daytime demand) }\end{array}$ & $\begin{array}{c}\text { Objective 2 } \\
\text { (nighttime demand) }\end{array}$ \\
\hline 0 & 382,411 & 421,583 \\
\hline 0.1 & 382,411 & 421,584 \\
\hline 0.2 & 382,411 & 421,582 \\
\hline 0.3 & 382,411 & 421,580 \\
\hline 0.4 & 382,411 & 421,589 \\
\hline 0.5 & 382,411 & 421,588 \\
\hline 0.6 & 382,411 & 421,587 \\
\hline 0.7 & 382,411 & 421,586 \\
\hline 0.8 & 382,411 & 421,585 \\
\hline 0.9 & 382,411 & 421,591 \\
\hline 1 & 379,203 & 416,842 \\
\hline
\end{tabular}

Service demand is represented by the 5,389 census blocks in the region (e.g., $|I|=5,389$ ), using nighttime $\left(a_{i}\right)$ and daytime $\left(\hat{a}_{i}\right)$ population estimates. The program currently supports nine facilities (e.g., $p=$ 9). There are 6,000 locations identified as potential facility sites (e.g., $|J|=6,000$ ). Road network travel is assumed with a suitable service coverage standard of 10 miles. ArcGIS is used to solve (6)-(11) implemented using (14) to account for nighttime demand $\left(a_{i}\right)$ and daytime demand $\left(\hat{a}_{i}\right)$ to be served. The 11 ArcGIS solution are summarized in Table 3 and Figure $2 \mathrm{a}$ for $w \in[0,1]$ in increments of 0.1 . For comparison purposes, the actual three non-dominated solutions derived using an exact approach combined with the constraint method within the Xpress optimization software package are given in Table 4 and also depicted in Figure 2a. This means there are actually 14 solutions summarized in Figure $2 \mathrm{a}$. Since the $w=0$ solution is more extreme (approximately $1 \%$ less than the optimum), the scale of the axes are such that it makes it difficult to see the range of ArcGIS solutions in Figure 2a (in fact, it appears that only three solutions are visible, which is not the case as there are actually 14 solutions). It is possible to zoom in (Figure $2 \mathrm{~b}$ ), ignoring the $w=0$ solution. Figure $2 \mathrm{~b}$ therefore highlights that, in this particular case, ArcGIS is not able to find the optimal non-dominated solutions, nor does it accurately reflect associated tradeoffs that are possible in distinguishing between the significance of service coverage to night and day populations.

Table 4. Actual non-dominated solutions.

\begin{tabular}{|l|c|c|}
\hline Solution & $\begin{array}{c}\text { Objective 1 } \\
\text { (daytime demand) }\end{array}$ & $\begin{array}{c}\text { Objective 2 } \\
\text { (nighttime demand) }\end{array}$ \\
\hline 1 & 382,411 & 421,614 \\
\hline 2 & 382,418 & 421,607 \\
\hline 3 & 382,921 & 421,488 \\
\hline
\end{tabular}

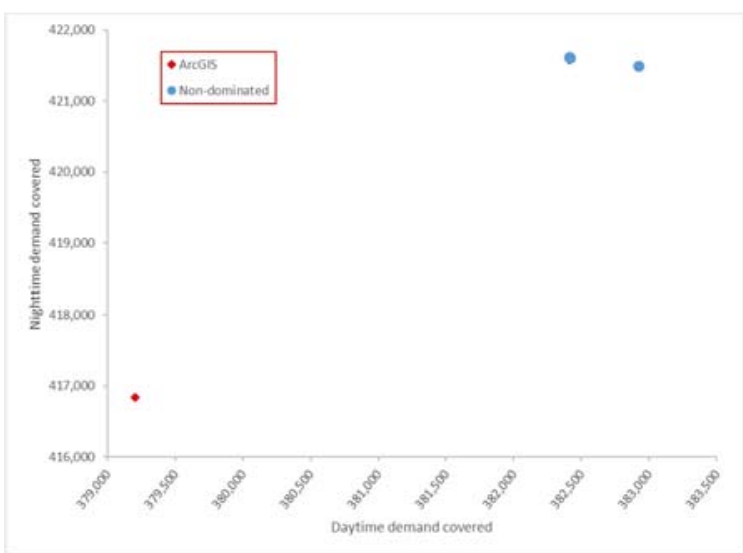

(a) Tradeoff showing all 11 ArcGIS solutions

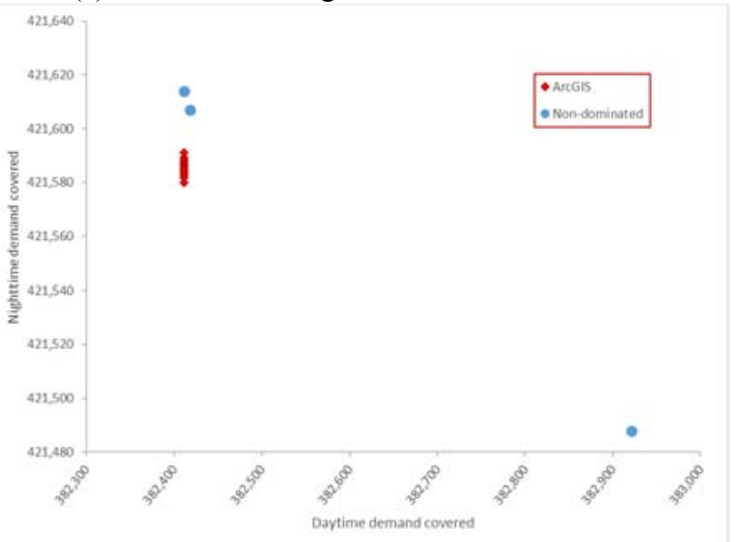

(b) Zoomed view omitting one ArcGIS solution $(w=0)$.

Figure 2. Comparison of ArcGIS heuristic results derived for the extension of the maximal covering location problem, (6)-(11).

\section{Discussion and Conclusions}

The range of application efforts relying on location analytics available through GIS is substantial, especially those that are based on spatial optimization for which reported application trends can be observed 
in Table 1. This is meaningful and important because planning, management, decision making and policy associated with location intelligence is widespread. However, it also raises concerns because many encountered location analytical methods in GIS are often ambiguous. This is both in terms of definition / specification as well as associated solution. Not only are there implications for reproducibility and replicability, but also they raise issues of reliability, appropriateness and validity, among others. As has been shown for ArcGIS and TransCAD, the description of location analytics often lacks specificity. Without advanced specialty knowledge and extensive empirical testing, such as that reported in $[27,38]$, it would be difficult to conclude exactly what a given location analytic approach is structuring and solving. Beyond definition, the heuristic solution approach too is lacking in important details regarding implementation. Add to this an inability for a user to control application parameters, such as number of times the heuristic is repeated or other convergence criteria, and there can be even less faith in the quality of obtained solutions.

The significance of definition, specification and solution quality for reproducibility and replicability is largely not understood. Since GIS software make it possible to reproduce and replicate because the methods are implemented in a general use manner, it would appear these are non-issues. However, heuristic performance is not consistent across applications. [27] observed application results within 1\% of optimal in some cases while in others over 7\%. [38] found solution quality to be even more varied, ranging up to $10 \%$ from optimal. For location analytics this creates potential confusion and added interpretation complexity because spatial pattern associated with any solution may vary greatly. Questions that arise are whether this variability is associated with poor solution quality or alternative / near optimal results.

The empirical results summarized in Figure 2 and Tables 3-4 highlight the replicability challenges when using location analytics available through GIS as methods can be used in extended ways. The extension of the maximal covering location problem, (6)-(11) (also referred to as the multiobjective facility location problem), can readily be carried out in ArcGIS and TransCAD. Indeed, this is not uncommon in practice. However, this require indirect processing using (14). Figure 2 and Tables 3-4 suggest, however, that derived results may be limited in various ways, both in terms of solution quality as well as diversity inherent to multi-objective problems.

In addition to the solution quality issue, there is also the consideration of computational effort. The required solution time for the heuristic results reported in Table 3 using ArcGIS was approximately 205 seconds for each instance of $w$. Solution time for the optimal results given in Table 4 required less than 30 seconds in each instance, though identification does involve evaluation of many problem instances (79 in this case). A personal computer (AMD Ryzen CPU $3900 \mathrm{X} 4.6 \mathrm{GHz}$ with 96GB RAM) running Windows 10 was relied upon to solve these problems. In theory, heuristics are appealing because they characteristically require less computational effort than exact methods. Unfortunately this was not the case with the application instances examined here.

Current software does not make it easy for novice users to understand what is happening for many commonly used GIS functions, particularly those supporting location intelligence. Do users understand the location analytic method they are applying? Do they understand the approach (heuristic) being used to derive results? Do they understand the associated assumptions and parameters of the method, and their impacts on obtained results, if any? What about the implications of underlying data uncertainty and frame dependency? Many of these issues are difficult to understand and address for experts in GIScience and location intelligence, so appreciation and considered application by non-experts is unrealistic. Nevertheless, location analytics in GIS continue to be used for deriving insights, management planning, decision making and policy evaluation/formulation (see Table 1), irrespective of replicability implications.

With increased movement to open source environments through Python and R, among others, access to GIS functionality continues to grow. So too does the availability of related spatial optimization approaches, mimicking methods found in commercial software packages. Along the lines discussed in this paper, issues of ambiguous definition, if any is even provided, and utilization of heuristics are common.

Perhaps the single most important step forward for location analytics in GIS is better communication. Methods must be defined in more rigorous ways, moving away from vague descriptions. Similarly, implemented solution techniques too must be precise in specification. However, beyond these two points, findings must simply be communicated in a manner that makes it clear for the point-and-click user that results may not be reproducible or replicable in various ways. There may indeed be some uncertainty, due to inappropriate method, non-optimality, parameters, assumptions, data, geographical representation, etc. This is critical considering that basic approaches can be subsequently extended. Any study or application effort likely has layers and layers of uncertainty, beginning with what is being done and permeating through produced results. Often there is 
little to no understanding of these complexities or their impacts. Of course, the significance depends on application context and the decisions being made, but reliability challenges are a concern for location intelligence.

\section{Acknowledgements}

The author would like to thank Seonga Cho, a graduate student at UCSB, for assistance in deriving the ArcGIS results reported in this paper.

\section{References}

[1] Alho, A. R., e Silva, J. D. A., de Sousa, J. P., \& Blanco, E. (2018). Improving mobility by optimizing the number, location and usage of loading/unloading bays for urban freight vehicles. Transportation Research Part D: Transport and Environment, 61, 3-18.

[2] Athira, G., Bahurudeen, A., Sahu, P. K., Santhanam, M., Nanthagopalan, P., \& Lalu, S. (2020). Effective utilization of sugar industry waste in Indian construction sector: A geospatial approach. Journal of Material Cycles and Waste Management, 1-13.

[3] Banerjee, S., Kabir, M. M., Khadem, N. K., \& Chavis, C. (2020). Optimal locations for bikeshare stations: A new GIS based spatial approach. Transportation Research Interdisciplinary Perspectives.

[4] Blanco, I., Loisi, R. V., Sica, C., Schettini, E., \& Vox, G. (2018). Agricultural plastic waste mapping using GIS. A case study in Italy. Resources, Conservation and Recycling, 137, 229-242.

[5] Brunsdon, C. (2016). Quantitative methods I: Reproducible research and quantitative geography. Progress in Human Geography, 40(5), 687-696.

[6] Church, R., \& ReVelle, C. (1974). The maximal covering location problem. Papers of the Regional Science Association, 32, 101-118.

[7] Church, R. L. (2001). Spatial optimization models. In International Encyclopedia of the Social and Behavioral Sciences, edited by L. Smelser and P. Baltes, 14811-14818. Elsevier.

[8] Church, R. L., \& Murray, A. (2018). Location Covering Models. Springer.

[9] Church, R. L., \& Murray, A. T. (2009). Business site selection, location analysis, and GIS. John Wiley \& Sons.

[10] Costa, F. R., Ribeiro, C. A. A. S., Marcatti, G. E., Lorenzon, A. S., Teixeira, T. R., Domingues, G. F., de Castro, N. L., dos Santos, A. R., Soares, V. P., Mota, P. H., \& de Almeida Telles, L. A. (2020). GIS applied to location of bioenergy plants in tropical agricultural areas. Renewable Energy, 153, 911-918.

[11] Fraser, A. M., Chester, M. V., \& Eisenman, D. (2018). Strategic locating of refuges for extreme heat events (or heat waves). Urban Climate, 25, 109-119.

[12] Gomez, D., Larsen, K., Burns, B. J., Dinh, M., \& Hsu, J. (2019). Optimizing access and configuration of trauma centre care in New South Wales. Injury, 50(5), 11051110 .

[13] Goodchild, M. F., \& Gopal, S. (eds) (1989). The Accuracy of Spatial Databases. CRC Press.

[14] Grubesic, T. H., \& Murray, A. T. (2002). Constructing the divide: Spatial disparities in broadband access. Papers in Regional Science, 81(2), 197-221.

[15] Irannezhad, E., Prato, C. G., \& Hickman, M. (2018). The effect of cooperation among shipping lines on transport costs and pollutant emissions. Transportation Research Part D: Transport and Environment, 65, 312-323.

[16] Kedron, P., Frazier, A. E., Trgovac, A. B., Nelson, T., \& Fotheringham, A. S. (2020). Reproducibility and replicability in Geographical Analysis. Geographical Analysis.

[17] Khan, M. M. U. H., Vaezi, M., \& Kumar, A. (2018). Optimal siting of solid waste-to-value-added facilities through a GIS-based assessment. Science of the Total Environment, 610, 1065-1075.

[18] Kocatepe, A., Ozguven, E. E., Horner, M., \& Ozel, H. (2018). Pet-and special needs-friendly shelter planning in South Florida: A spatial capacitated p-median-based approach. International Journal of Disaster Risk Reduction, 31, 1207-1222.

[19] Lane, B., Shaffer, B., \& Samuelsen, S. (2020). A comparison of alternative vehicle fueling infrastructure scenarios. Applied Energy, 259.

[20] Lemire, P. O., Delcroix, B., Audy, J. F., Labelle, F., Mangin, P., \& Barnabé, S. (2019). GIS method to design and assess the transportation performance of a decentralized biorefinery supply system and comparison with a centralized system: case study in southern Quebec, Canada. Biofuels, Bioproducts and Biorefining, 13(3), 552-567.

[21] Medrano, F. A., \& Church, R. L. (2014). Corridor location for infrastructure development: A fast bi-objective shortest path method for approximating the Pareto frontier. International Regional Science Review, 37(2), 129-148.

[22] Mishra, S., Sahu, P. K., Sarkar, A. K., Mehran, B., \& Sharma, S. (2019). Geo-spatial site suitability analysis for development of health care units in rural India: Effects on habitation accessibility, facility utilization and zonal equity in facility distribution. Journal of Transport Geography, 78, 135-149.

[23] Murray, A. T. (2007). Optimization. In Encyclopedia of Geographic Information Science, edited by K. Kemp, 333-334. Sage.

[24] Murray, A. T. (2018). Complexities in spatial center derivation. Transactions in GIS, 22(6), 1335-1350.

[25] Murray, A. T. (2019). Geocomputation and spatial analytics. In GeoComputation 2019.

[26] Murray, A. T. (2020). Contemporary optimization application through geographic information systems. Omega.

[27] Murray, A. T., Xu, J., Wang, Z., \& Church, R. L. (2019). Commercial GIS location analytics: capabilities and performance. International Journal of Geographical Information Science, 33(5), 1106-1130.

[28] National Academies of Sciences, Engineering, Medicine (2019). Reproducibility and replicability in science. National Academies Press. 
[29] Openshaw, S., \& Taylor, P. J. (1981). The modifiable areal unit problem. In Quantitative Geography: A British View, edited by N. Wrigley and R. Bennett, 60-69. Routledge and Kegan Paul.

[30] Rey, S. J. (2018). Code as text: Open source lessons for geospatial research and education. In Geocomputational Analysis and Modeling of Regional Systems, edited by J.C. Thill and S. Dragicevic, 7-21. Springer.

[31] Romanillos, G., \& García-Palomares, J. C. (2018). Accessibility to Schools: Spatial and Social Imbalances and the Impact of Population Density in Four European Cities. Journal of Urban Planning and Development, 144(4), 04018044.

[32] Schilling, D. A., Revelle, C., Cohon, J., \& Elzinga, D. J. (1980). Some models for fire protection locational decisions. European Journal of Operational Research, 5(1), 1-7.

[33] Schröder, M., \& Cabral, P. (2019). Eco-friendly 3D-Routing: A GIS based 3D-Routing-Model to estimate and reduce $\mathrm{CO} 2$-emissions of distribution transports. Computers, Environment and Urban Systems, 73, 40-55.

[34] Teixeira, T. R., Ribeiro, C. A. A. S., dos Santos, A. R., Marcatti, G. E., Lorenzon, A. S., de Castro, N. L. M., Domingues, G. F., Leite, H. G., Mota, P. H. S., de Almeida Telles, L. A., \& da Silva Vieira, R. (2018). Forest biomass power plant installation scenarios. Biomass and Bioenergy, $108,35-47$.

[35] Tobler, W. R. (1989). Frame independent spatial analysis. In The Accuracy of Spatial Databases, edited by M.F. Goodchild and S. Gopal, 115-122. Taylor and Francis. [36] Tong, D., \& Murray, A. T. (2012). Spatial optimization in geography. Annals of the Association of American Geographers, 102(6), 1290-1309.

[37] Trindade, A., Teves-Costa, P., \& Catita, C. (2018). A GIS-based analysis of constraints on pedestrian tsunami evacuation routes: Cascais case study (Portugal). Natural Hazards, 93(1), 169-185.

[38] Xu, J., Murray, A., Wang, Z., \& Church, R. (2020). Challenges in applying capacitated covering models. Transactions in GIS, 24(2), 268-290. 